\title{
Animales de compañía en la UE: Identificación y registro
}

Keywords: Animales de compañía; perros; gatos; pasaporte de viaje UE para animales de compañía

La UE tiene una prolija y abundante legislación en materia de Bienestar Animal, referida sobre todo a animales de producción, transporte y experimentación. Sin embargo, quedan por regular los animales de compañía, de forma singular y obligatoria para todos los Estados Miembros. En este sentido, el Parlamento Europeo en una reciente moción de 25 de febrero de 2016 relativa a la identificación y registro de los animales de compañía, ha dado un importante primer paso.

Durante los últimos años, se han dictado medidas para regular algunos aspectos derivados de la convivencia con los animales de compañía que, en la UE representan un importante volumen de más 100

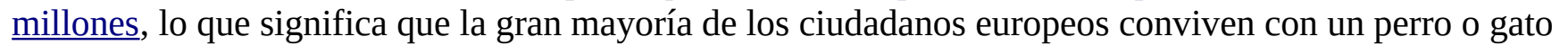
aunque la proporción, dependiendo de los países, es muy variable. Así mismo, son muy diferentes las normativas de los Estados Miembros, por lo que construir una normativa común a toda la UE ha encontrado, particularmente en este tema, resistencias casi imposibles de vencer[1].

En lo relativo al registro e identificación de animales de compañía, los datos que suministran los Estados Miembros presentan diferencias llamativas. En principio, todos los Estados de la UE tienen implantado un sistema nacional de registro e identificación incompatible con los de los restantes Estados Miembros. Incluso 6 países de la UE carecen de sistema identificador y de registro, a saber: la República Checa, Finlandia, Polonia, Alemania (sólo algunos Länder), Irlanda (previsto para el 2016) y el Reino Unido, donde Escocia e Inglaterra tienen prevista la implantación en la primavera de 2016. En el caso de España, la identificación y registro de los animales de compañía son competencia de las distintas Comunidades Autónomas con lo que, en nuestro caso, tenemos 17 registros distintos, no compatibles entre sí, que se suman al "caos" del resto de los países europeos en este tema. Se han promovido algunas iniciativas particulares, tendentes a armonizar y unificar los criterios relativos al registro e identificación de animales como EUROPETNET, o PETTMAX, pero no han resultado totalmente satisfactorias, ni han sido aceptadas por los gobiernos de los países de la UE.

La estrategia que la UE ha diseñado en las últimas décadas, en tema de animales de compañía, ha partido del reconocimiento de dos problemas que nos afectan a todos de manera grave. Se trata del abandono de perros y gatos, así como del tráfico ilegal de cachorros [2], particularmente procedentes de los países del Este. Dicha estrategia, se dirige a advertir del peligro que tales prácticas representan para la salud de todos (animales y humanos) y a concienciar a la sociedad de los hábitos y conductas que configuran lo que se suele denominar tenencia responsable. Por ello, se han fomentado comisiones[3], reuniones de expertos[4] y campañas, para encontrar puntos de anclaje comunes desde donde impulsar políticas públicas que permitan salir de lo que viene constituyendo, sobre todo en los últimos años, un problema insoluble hasta el momento.

Un estudio llevado a cabo por la CE, ponía de relieve, ya en el año 2014, que el comercio ilegal de gatos y perros representa una ganancia aproximada de 1,3 billones de Euros. En concreto, la importación de perros supone anualmente 21 millones de Euros (2014) y de 3 millones de Euros en lo concerniente al tráfico de gatos (2014). El dato más sorprendente es que sólo el 13\% de las compras de animales de compañía proceden de criadores profesionales[5]. El Dr. Andrea Gavinelli subrayaba que "algunos países 

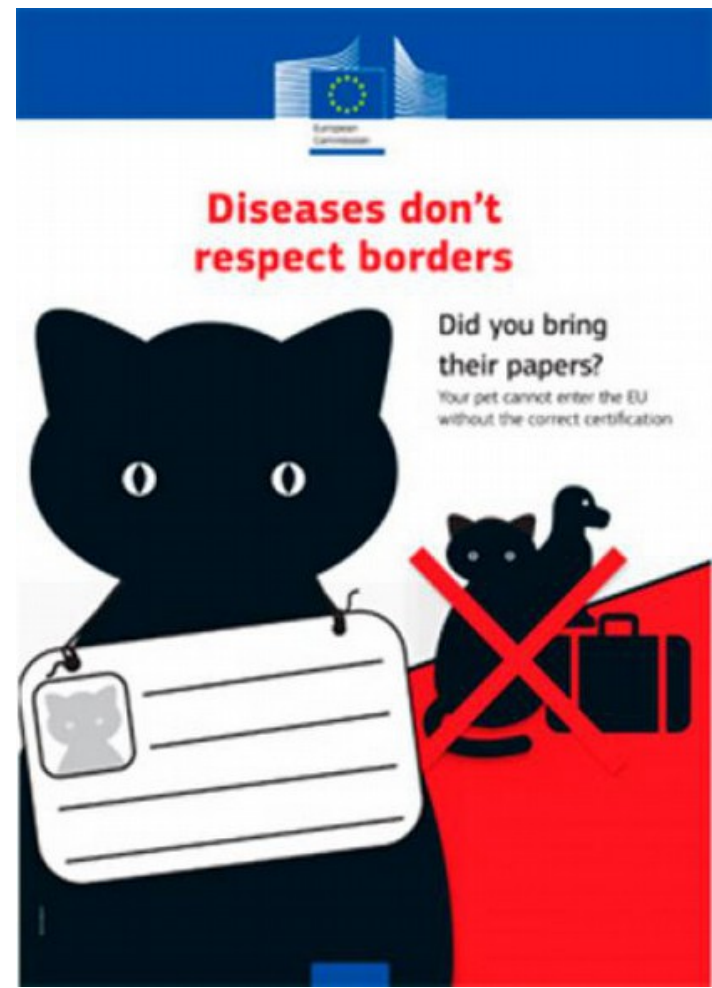

europeos, como Polonia, Rumanía, Eslovaquia, Hungría o España, carecen de una definición legal de criador profesional"[6]. Son datos contrastados, que el tráfico ilegal de animales ocupa en Europa el tercer lugar tras el tráfico de drogas y de armas, por lo que la trazabilidad de los animales en tránsito, podría evitar tales prácticas infames y que el producto de estas ventas ilegales de grupos organizados, fuera a parar al mercado negro. Las denuncias que en este sentido se hacen, al menos en España, han conducido a que la Guardia Civil haya conseguido desmantelar establecimientos ilegales de cría y a rescatar a los cachorros[7], que estaban dispuestos para ser distribuidos a tiendas y a particulares, pues la mayoría de estas ventas se hacen a través de internet.

La primera de las iniciativas que ha encontrado un consenso general ha sido la de crear un sistema unificado de registro e identificación de los animales de compañía, en línea con lo dispuesto en el artículo 13 TFUE, respecto a la consideración de los animales como seres sintientes ("sentient beings"). Es evidente que la implantación efectiva de tal sistema, facilitaría la trazabilidad de los animales de compañía y la atribución de responsabilidades, en los casos señalados de abandono y de tráfico ilegal que, además, envuelven a criaturas tan vulnerables, como son los cachorros, en situaciones de extremo maltrato.

En este sentido una de las campañas recientes de la UE, ha puesto el acento en la necesidad de proveer de un pasaporte reglado a los animales de compañía en tránsito por Europa (Pet Travel Scheme)[8], como medio de evitar la propagación de enfermedades (zoonosis) transmitidas por los animales[9]. Es claro que el trasfondo de la campaña es alertar sobre la salud pública, pero, cada vez más se habla de "One Health", para hacer referencia no sólo a la salud humana sino también a la de los animales. Un control estricto de la identidad, procedencia, vacunaciones y pertenencia de los animales que circulan por territorio de la UE, sería uno de los elementos disuasorios más eficaces para combatir conductas claramente punibles, como lo es también el abandono, que en la reciente reforma del C.Penal español ha pasado a convertirse de falta en delito[10].

Estas y otras razones, están expuestas de forma pormenorizada en el preámbulo de la reciente moción conjunta dictada por el Parlamento de la UE, en fecha de marzo[11]. El texto aprobado, contiene 11 motivaciones y recomendaciones en las que vale la pena detenerse, pues denotan las carencias de la legislación existente en tema de animales de compañía en el espacio UE, ya señaladas anteriormente, pero también subraya en la primera conclusión el importante papel que los animales de compañía desempeñan en la vida de millones de individuos y de familias europeas[12]. Por ello, como prevención de los abusos mencionados, se dispone en el nº la obligatoriedad y registro de los animales de compañía y la creación de una base de datos compatible para todos los Estados Miembros de la UE, en la que se asegure la trazabilidad de los animales y la punibilidad de las conductas que contravengan las líneas básicas de bienestar que como seres sintientes merecen. Este es, a mi juicio, un buen inicio para que los organismos europeos logren una legislación marco para los animales de compañía, cada día mas importantes en la UE. 
[1] A este propósito, CALLISTO Project.

[2] Vid. el informe relativo al tráfico ilegal de cachorros, correspondiente al año 2014, "Illegal Puppy Trade Report of 2014".

[3] Vid. 1st. EU Conference on dogs and cats "Building a Europe that cares for Companion Animals, 28.10.2013; 2nd. EU

Conference on the Welfare on dogs and cats involved in commercial practices, Bruselas 2 de Noviembre de 2015.

[4] EU Canine and Feline Traceability Expert Group en el marco de los proyectos CAROdog y CAROcat (www.carocat.eu), bajo el impulso decisivo de la Fundación Vier-Pfoten, una de las entidades colaboradoras del Máster en Derecho Animal y Sociedad de la UAB desde el inicio.

[5] este propósito, el informe de Carodog.

[6] Vid. la transcripción de las palabras del Dr. A. Gavinelli, en la 2nd. Conference on the welfare on dogs and cats involved in commercial practices.

[7] Vid. el último Boletín de Prensa de la Guardia civil.

[8] Pet Travel Scheme introducido por el Reglamento (EU) del Parlamento y del Consejo No 576/2013, de 12 de Junio de 2013.

[9] Vid la reciente ley europea de salud animal, Animal Health Law. New EU Regulation on transmissible animal diseases. June 2015.

[10] RIOS CORBACHO, JM, Comentario en relación al maltrato de animales en la reforma del nuevo Código Penal español (LO1/2015),; REQUEJO CONDE, MC, El delito de maltrato a los animales tras la reforma del Código Penal por la Ley Orgánica $1 / 2015$, de 30 de marzo.

[11] European Parliament resolution on the harmonisation of identification and registration requirements for pet animals for the purposes of the protection of public and animal health (2016/2540(RSP).

[12] Ibidem, 1." Underlines the positive contribution that pet animals make to the lives of millions of individual owners and families across the EU, and reaffirms that owners should be able to travel with their pets in a safe, controlled manner throughout the Union" 